\title{
Excitons and Many-Electron Effects in the Optical Response of Single-Walled Boron Nitride Nanotubes
}

\author{
Cheol-Hwan Park, Catalin D. Spataru, and Steven G. Louie \\ Department of Physics, University of California at Berkeley, Berkeley, California 94720 \\ Materials Sciences Division, Lawrence Berkeley National Laboratory, Berkeley, California 94720
}

(Dated: November 6, 2018)

\begin{abstract}
We report first-principles calculations of the effects of quasiparticle self-energy and electron-hole interaction on the optical properties of single-walled BN nanotubes. Excitonic effects are shown to be even more important in $\mathrm{BN}$ nanotubes than in carbon nanotubes. Electron-hole interactions give rise to complexes of bright (and dark) excitons, which qualitatively alter the optical response. Excitons with binding energy larger than $2 \mathrm{eV}$ are found in the $(8,0) \mathrm{BN}$ nanotubes. Moreover, unlike the carbon nanotubes, theory predicts that these exciton states are comprised of coherent supposition of transitions from several different subband pairs, giving rise to novel behaviors.
\end{abstract}

Boron nitride nanotubes (BNNTs) are isoelectronic to carbon nanotubes (CNTs); however, their electronic properties are quite different. Whereas carbon nanotubes are metals or semiconductors with different size bandgaps depending on diameter and chirality [1], BN nanotubes are wide gap insulators 2, 3]. Although BNNTs have been synthesized since 1995 [4], only recently optical measurement on single-walled BNNTs has been performed [5]. Theoretical calculations [6, 7], as well as experiments [8, 9, 10], have shown that excitonic effects dramatically alter the behavior of the optical response of single-walled CNTs. For the BNNTs, these effects are expected to be even more important due to the wide band gap nature of BNNTs.

Experimentally it is found that BN nanotubes favor zigzag structure in current synthesis processes 11]. Thus, we focus our study on the zigzag tubes. Our calculations on the $(8,0)$ single-walled BNNT show that, indeed, many-electron effects lead to the formation of strongly bound excitons of multi-band character with extraordinarily large binding energies, which dramatically change its optical absorption spectrum.

To compute the optical response, we use the method of Rohlfing and Louie [12] in which electron-hole excitations and optical spectra are calculated from first principles in three steps. First, we treat the electronic ground state with ab initio pseudopotential density-functional theory (DFT) 13. . Second, we obtain the quasiparticle energies $E_{n \mathbf{k}}$ within the $G W$ approximation for the electron selfenergy $\Sigma[14$ by solving the Dyson equation:

$$
\left[-\frac{\nabla^{2}}{2}+V_{i o n}+V_{\text {Hartree }}+\Sigma\left(E_{n \mathbf{k}}\right)\right] \psi_{n \mathbf{k}}=E_{n \mathbf{k}} \psi_{n \mathbf{k}} \text {. }
$$

Finally, we calculate the coupled electron-hole excitation energies and optical spectrum by solving the BetheSalpeter (BS) equation of the two-particle Green's function [12, 15]:

$\left(E_{c \mathbf{k}}-E_{v \mathbf{k}}\right) A_{v c \mathbf{k}}^{S}+\sum_{\mathbf{k}^{\prime} v^{\prime} c^{\prime}}\left\langle v c \mathbf{k}\left|K^{e h}\right| v^{\prime} c^{\prime} \mathbf{k}^{\prime}\right\rangle A_{v^{\prime} c^{\prime} \mathbf{k}^{\prime}}^{S}=\Omega^{S} A_{v c \mathbf{k}}^{S}$, where $A_{v c \mathbf{k}}^{S}$ is the exciton amplitude, $K^{e h}$ is the electronhole interaction kernel, and $|c \mathbf{k}\rangle$ and $|v \mathbf{k}\rangle$ are the quasielectron and quasihole states, respectively.
The DFT eigenvalues and wave functions were obtained within the local density approximation (LDA) 13. using a plane-wave basis [16] with an energy cutoff of 100 Ry. ab initio Troullier-Martins pseudopotentials 17] in the Kleinman-Bylander form [18] were used (with cutoff $r_{c}$ for boron and nitrogen of $0.79 \AA$ and $0.63 \AA$, respectively). For convergent results to better than $0.05 \mathrm{eV}$, up to $32 \mathrm{k}$ points in the one-dimensional Brillouin zone were used for the $G W$ calculations and for solving the BS equation. All calculations were carried out in a supercell geometry with a wall-to-wall intertube separation of $9.5 \AA$ to mimic isolated tubes, together with a truncated Coulomb interaction in the radial direction in order to eliminate unphysical interactions between periodic images on the different tubes. As shown in Ref. 19], it is important to truncate the Coulomb interaction because these unphysical interactions would increase the effective screening in the system and hence reduce both the selfenergy correction and the exciton binding energy. Because of depolarization effects in nanotubes [20], strong optical response is only observed for light polarized along the tube axis $(\hat{z})$. We consider here only this polarization.

Figure 1(a) shows the quasiparticle energy corrections to the LDA energy eigenvalues. First, we note that these corrections are quite large, in comparison to those for bulk hexagonal BN (h-BN) and SWCNTs. The quasiparticle corrections open the LDA gap of bulk h-BN by $\approx 1.58 \mathrm{eV}$ near zone center or the $\Gamma$-point [21], while the gap opening in the $(8,0)$ SWBNNT near the $\Gamma$-point is $\approx 3.25 \mathrm{eV}$. This is a consequence of enhanced Coulomb interaction in reduced dimension 7]. Also, due to its larger gap, which weakens screening, the quasiparticle corrections to the gap in the $(8,0)$ SWBNNT are larger than those for a similar SWCNT (which are $\approx 1.15 \mathrm{eV}$ near the $\Gamma$-point [7]). Second, the quasiparticle corrections have a complex band- and energy-dependence, so for accurate results they cannot be obtained by a simple scissor shift operation. The corrections depend on the character of the wavefunction. For example, states of the fourth lowest conduction band in the LDA bandstructure 

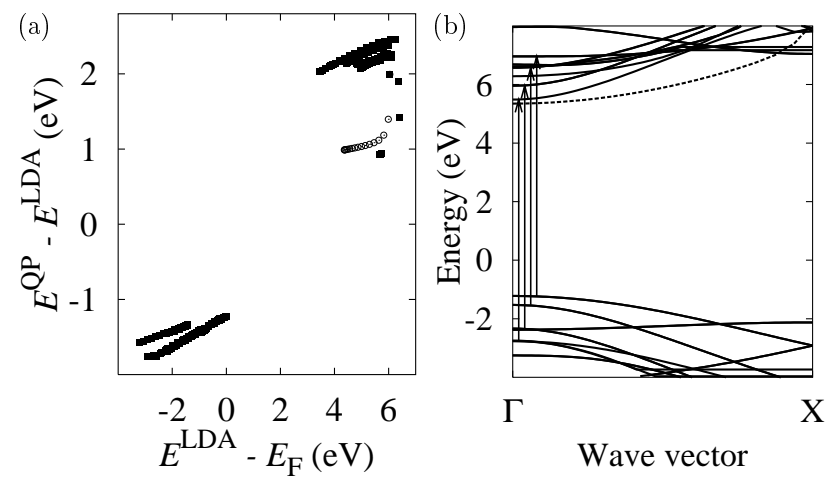

FIG. 1: Difference between the $G W$ quasiparticle energy and the LDA Kohn-Sham eigenvalue plotted as a function of the energy of the states (a) and quasiparticle band-structure (b) for the $(8,0)$ SWBNNT. Empty circles in (a) and the dashed line in (b) show the nearly-free-electron tubule states.

are localized inside the tube, and are nearly free-electronlike states. These tubule states form a separate branch in the quasiparticle correction diagram with significantly smaller corrections. Figure (b) depicts the quasiparticle bandstructure of the $(8,0)$ SWBNNT. The arrows indicate the optically allowed interband transitions between four pairs of bands which give rise to the lowest-energy peak structures in the non-interacting optical spectrum in Fig. 2 (The fourth lowest energy conduction band in the LDA bandstructure becomes the lowest energy conduction band in the quasiparticle bandstructure.)

Figure2 depicts the optical absorption spectrum calculated with and without electron-hole interaction effects. The plotted quantity is the imaginary part of the calculated dielectric susceptibility, $\chi=(\epsilon-1) / 4 \pi$, multiplied by the cross-sectional area of the supercell perpendicular to the tube axis. This quantity $\alpha$, as defined above, gives the polarizability per single tube in units of $\mathrm{nm}^{2}$; so the susceptibility of an experimental sample containing a density of $n$ infinitely long tubes per unit area may be obtained as $\chi=n \alpha$. The absorption profile changes dramatically when the electron-hole interaction is taken into account. We use the label $I, I^{\prime}$, and $I I$ to denote the excitons that give rise to the absorption peaks in the figure. Subscripts 1, 2, 3, and 4 refer to the ground, first-excited, second-excited, and third-excited states of a particular bright exciton series, respectively. We observe a strong absorption peak at $5.72 \mathrm{eV}$, which corresponds to a bound exciton $\left(I_{1}\right)$ with a binding energy of $2.3 \mathrm{eV}$. The area under this peak, which may be used in comparing with experiment, is $0.87 \mathrm{~nm}^{2} \mathrm{eV}$. Excitons $I_{1}$ and $I_{1}^{\prime}$ are different states, made up of transitions from the same set of four pairs of valence and conduction subbands of the $(8,0) \mathrm{BNNT}$, all of which have similar quasiparticle transition energies from $8.1 \mathrm{eV}$ to $8.3 \mathrm{eV}$ (See arrows in Fig. प(b)). These transitions are coupled strongly to each other by the electron-hole interaction to form the

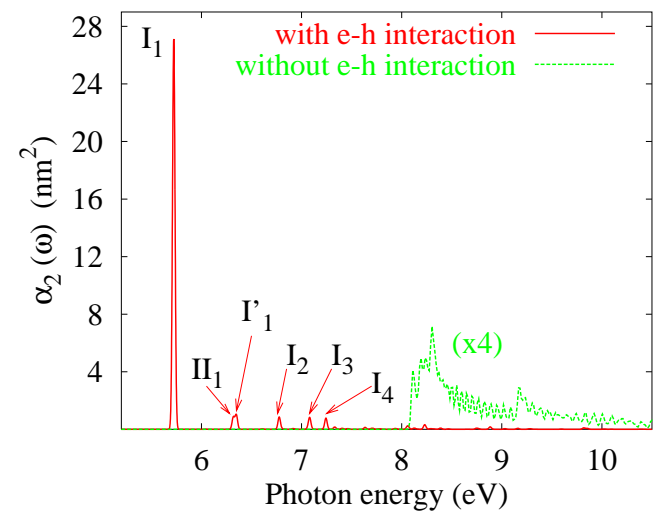

FIG. 2: (color online). Absorption spectra of the $(8,0)$ SWBNNTs. The imaginary part of the polarizability per tube $\alpha_{2}(\omega)$ is given in unit of $\mathrm{nm}^{2}$. (See text.) The spectra are broadened with a Gaussian of $0.0125 \mathrm{eV}$.

lowest optically active states (the singly-degenerate $I_{1}$ and doubly-degenerate $I_{1}^{\prime}$ ). This behavior is very different from the $(8,0)$ SWCNT in which the exciton states are composed mainly of transitions between a single pair of quasiparticle bands.

The mixing of transitions of different subbands alters the electron-hole wavefunction - localizing further the electron amplitude with respect to the hole position in real space and making it deviate from a 1-dimensional behavior with spatial variations in directions perpendicular to the tube axis. Figure 3(a) shows the isosurface plots of the electron distribution $\left|\Phi\left(\mathbf{r}_{e}, \mathbf{r}_{h}\right)\right|^{2}$ with the hole position $\mathbf{r}_{h}$ fixed (the black star in the figure) for the first bound exciton $\left(I_{1}\right)$. Figure 3(b) quantifies the electronhole correlation for this state by plotting $|\Phi|^{2}$ along the tube axis after integrating out the electron coordinates in the perpendicular plane (the hole position is set at zero). The position of the peaks in Fig [3(b) corresponds to the position of plane of boron atoms, i.e., the photoexcited electron is localized on the boron atoms near the hole. Thus, as expected, the photo-excitation process corresponds to a transfer of electron from nitrogen atoms to nearby boron atoms; but the resulting electron and hole amplitudes are strongly correlated with an extent of only a few inter-atomic distances. Figure 3(c) is a cross-sectional plot, showing the excited electron probability distribution in a plane that is perpendicular to the tube axis and contains the hole as well as other nitrogen atoms.

As a comparison to carbon nanotubes, Fig. B(d)-(f) shows similar quantities as in Fig. 3(a)-(c) but for the first bright bound exciton in the $(8,0)$ SWCNT []]. In the figure, the hole is fixed slightly above a carbon atom. The exciton in the $(8,0)$ SWBNNT is significantly more tightly bound than that in the $(8,0)$ SWCNT and cannot really be viewed as a $1-\mathrm{D}$ object. The root-mean-square size of the exciton along the tube axis is $3.67 \AA$ for the 

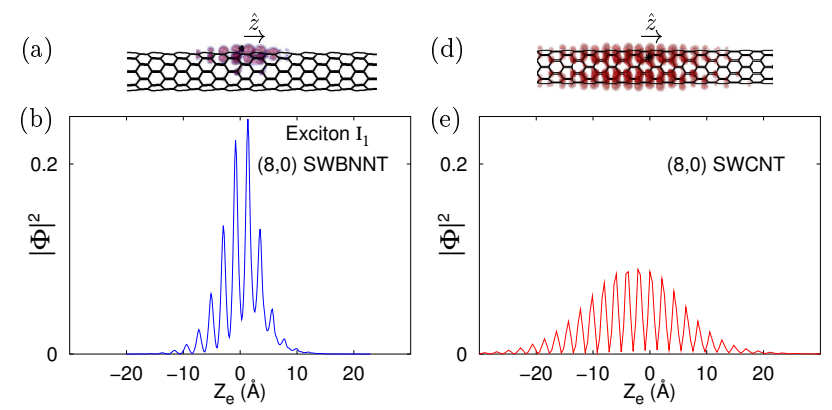

(c)
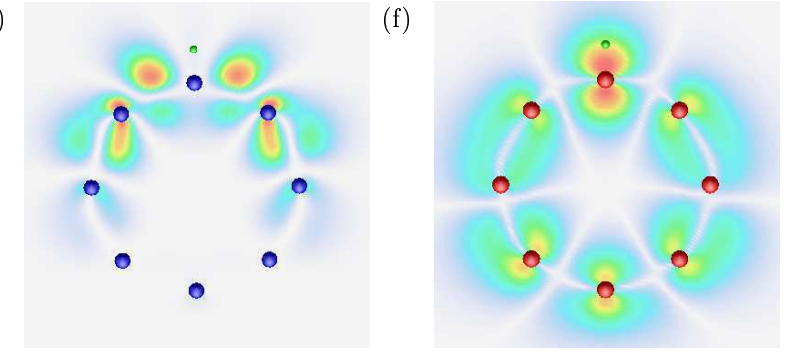

FIG. 3: (color online). (a)-(c): Wavefunction of the lowest energy bright exciton of the $(8,0)$ SWBNNT. (a) Isosurface plot of electron probability distribution $\left|\Phi\left(\mathbf{r}_{e}, \mathbf{r}_{h}\right)\right|^{2}$ with the hole fixed at the position indicated by black star. (b) $\left|\Phi\left(\mathbf{r}_{e}, \mathbf{r}_{h}\right)\right|^{2}$ averaged over tube cross section. Hole position is set at zero. (c) $\left|\Phi\left(\mathbf{r}_{e}, \mathbf{r}_{h}\right)\right|^{2}$ evaluated on a cross-sectional plane of the tube. (d)-(f): Wavefunction of the lowest energy bright exciton of the $(8,0)$ SWCNT. Plotted quantities are similar to those in (a)-(c).

$(8,0)$ SWBNNT and $8.59 \AA$ for the $(8,0)$ SWCNT, and their binding energies are $2.3 \mathrm{eV}$ and $1.0 \mathrm{eV}$, respectively. This difference in behavior is due to the wide bandgap and weaker screening in SWBNNT. Also, we note that while the binding energy of the excitons in the bulk h$\mathrm{BN}$ is only $0.7 \mathrm{eV}[22,23]$, the binding energy in the $(8,0)$ SWBNNT is more than three times larger.

Figures $4(\mathrm{a})$ and $4(\mathrm{~b})$ show similar quantities as in Fig. 3(b) for the excitons $I_{1}^{\prime}$ and $I_{2}$. For exciton $I_{1}^{\prime}$, the electron is less tightly bound to the hole than in exciton $I_{1}$. The state $I_{2}$, which is an excited state of exciton $I_{1}$, is also more diffuse than $I_{1}$ and the electron amplitude is not at a maximum near the hole which is the case for $I_{1}$ (Fig. 3(b)). We also note that, for the $(8,0)$ SWBNNT, there are numerous dark excitons distributed rather uniformly in energy below and among the bright excitons shown in Fig. 22 The energy of the lowest doubly-degenerate bound dark exciton is at $4.63 \mathrm{eV}$. This dark exciton is made up of transitions from the highest valence band to the lowest conduction band (the NFE tubule state) in the quasiparticle bandstructure, and has a binding energy of $1.94 \mathrm{eV}$ with respect to these interband transition energies.

The various lowest-energy exciton states (for both bright and dark excitons) derived from the various different sets of interband transitions, on the average, have a large binding energy of about $1.9 \mathrm{eV}$. However, the
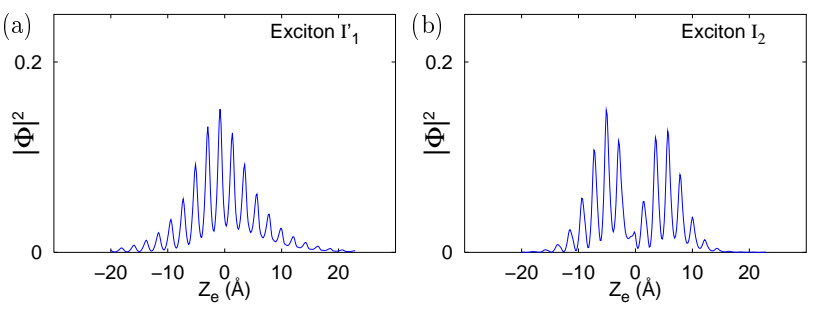

FIG. 4: (color online). Wavefunctions of excitons of the $(8,0)$ SWBNNT. Plotted quantities are similar to those in Fig. 3(b).

binding energy of the first bright exciton is $2.3 \mathrm{eV}$. We ascribe this extra binding energy of about $0.4 \mathrm{eV}$ to the fact that four different sets of interband transitions are strongly coupled in forming the first bright exciton $I_{1}$. This strong coupling mixes states from the different transitions, splits the excitation energy levels, and increases the binding energy of the final lowest-energy exciton.

Lauret et. al. [5] recently measured the optical properties of samples containing SWBNNTs and observed three absorption peaks at $4.45,5.5$, and $6.15 \mathrm{eV}$, respectively. These authors assigned the $4.45 \mathrm{eV}$ peak to originate from a bound exciton state. In our calculation, the first strong absorption peak is at $5.72 \mathrm{eV}$ which is about 1.3 $\mathrm{eV}$ blue shifted from the observed peak. A direct comparison between theory and experiment however should not be made. The average diameter of the tubes measured in the experiment is $1.4 \mathrm{~nm}$ while the diameter of the relaxed $(8,0)$ SWBNNT in our work is $0.65 \mathrm{~nm}$. Also, in the experiment, there would be tubes with different chiralities.

It is unlikely that the large discrepancy of $1.3 \mathrm{eV}$ is due to differences in diameter or chirality. At the LDA level, which neglects both self-energy corrections and excitonic effects, the position of the first broad absorption peak for SWBNNTs with diameter of $0.48 \mathrm{~nm}$ for a $(6,0)$ tube and $2.13 \mathrm{~nm}$ for a $(27,0)$ tube is at $4.8 \mathrm{eV}$ and $5.6 \mathrm{eV}$, respectively 24]. The difference between them is only $0.8 \mathrm{eV}$. Furthermore, assuming same chirality, larger diameter tubes tend to have their first absorption peak at higher energy. For these reasons, it is difficult to explain the $1.3 \mathrm{eV}$ discrepancy. The calculated first peak position for the $(8,0)$ tube is however rather close to the observed $5.5 \mathrm{eV}$ peak. Also, the observed difference between the second and the third absorption peak position in the experiment is $0.65 \mathrm{eV}$, very close to the difference between the first and the second absorption peaks in our calculation which is $0.62 \mathrm{eV}$. But the difference between the first and the second peak position in the experiment is $1.05 \mathrm{eV}$. Another difference is that the theory is for an isolated tube, while experimentally the tubes are surrounded by a dielectric medium which can modify the excitation energies. We thus suspect that the observed second peak at $5.5 \mathrm{eV}$ may be due to an exciton, corre- 
sponding in nature to our first absorption peak at 5.72 $\mathrm{eV}$. However, an additional complication is the existence of the many dark excitons, which could be made active by external perturbations. The exact interpretation for the experimental observations can therefore only be made after further experimental and theoretical studies.

For SWCNTs, the effect of the surrounding dielectric medium on the optical spectrum is expected to be small (even though it can be important for the exciton binding energy) due to an almost cancellation between the quasiparticle self-energy correction and the binding energy of excitons 7]. For SWBNNTs, screening by external medium would be more important because intrinsic screening is much weaker. In particular, in isolated SWBNNTs, we find that the energy difference between the quasiparticle self-energy correction and the binding energy of the exciton is large (about $0.9 \mathrm{eV}$ ). In the presence of a dielectric medium, we expect this energy difference to decrease, which would result in a decrease in the excitation energies.

Among previous theoretical works on the optical properties of BNNTs, Guo and Lin [24] carried out calculations at the LDA-RPA level without considering manyelectron effects. Their optical absorption spectra are qualitatively very different from the present final results. From their results for the $(6,0)$ and $(9,0)$ tubes (showing broad absorption peaks at $4.8 \mathrm{eV}$ and $5.0 \mathrm{eV}$, respectively), we can deduce a peak position for the $(8,0)$ tube to be near $4.9 \mathrm{eV}$, as we find in our LDA-RPA level calculation. The first peak position in Fig. 2] with electron-hole interaction included is blue shifted by about $0.9 \mathrm{eV}$ from that of the LDA-RPA calculation. Ng and Zhang [25], on the other hand, used a time-dependent, localized-densitymatrix approach based on a semiempirical Hamiltonian to compute the optical properties of SWBNNTs. In their work, the absorption spectrum of $(8,0)$ BNNT has a very broad first peak at an even higher energy than ours, at near $6.2 \mathrm{eV}$.

In summary, we have done calculation on the zigzag $(8,0)$ SWBNNT to study the effects of many-electron interactions on its optical response. The $G W$ corrections to the quasiparticle excitation energies of the SWBNNTs are significantly larger than those for SWCNTs or bulk h-BN. Also, the quasiparticle energy corrections are found to be complicated so that interpolation by a simple scissor shift operation is not a good scheme for accurate calculation. Theory predicts that, unlike the noninteracting case, the absorption spectrum of the $(8,0)$ SWBNNT is dominated by a huge peak at $5.72 \mathrm{eV}$, due to an exciton with a large binding energy of $2.3 \mathrm{eV}$. This exciton state is made up of optically-allowed transitions between four different pairs of subbands. Moreover, an intricate set of dark excitons is found to exist. Self-energy and electron-hole interaction effects therefore are even more important in the optical response of the SWBNNTs than in the SWCNTs.

This work was supported by the NSF under Grant No. DMR04-39768, and by the Director, Office of Science, Office of Basic Energy Sciences, Division of Materials Sciences and Engineering, U.S. Department of Energy under Contract No. DE-AC03-76SF00098. Computational resources were provided by NPACI and NERSC.

[1] R. Saito, G. Dresselhaus, and M.S. Dresselhaus, Physical Properties of Carbon Nanotubes (Imperial College, London, 1998).

[2] A. Rubio, J.L. Corkill, and M.L. Cohen, Phys. Rev. B 49, R5081 (1994).

[3] X. Blase, A. Rubio, S.G. Louie, and M.L. Cohen, Europhys. Lett. 28, 335 (1994).

[4] N.G. Chopra, J. Luyken, K. Cherry, V.H. Crespi, M. Cohen, S.G. Louie, and A. Zettl, Science 269, 966 (1995).

[5] J.S. Lauret, R. Arenal, F. Ducastelle, and A. Loiseau, M. Cau, B. Attal-Tretout, E. Rosencher, and L. GouxCapes, Phys. Rev. Lett. 94, 037405 (2005).

[6] T. Ando, J. Phys. Soc. Japan 66, 1066 (1996).

[7] C.D. Spataru, S. Ismail-Beigi, L.X. Benedict and S.G. Louie, Phys. Rev. Lett. 92, 077402 (2004).

[8] F. Wang, G. Dukovic, L.E. Brus, and T.F. Heinz, Science 208, 838 (2005).

[9] Y.-Z. Ma, L. Valkunas, S.L. Dexheimer, S. M. Bachilo, and G. R. Fleming, Phys. Rev. Lett. 94, 157402 (2005).

[10] Y.-Z. Ma, L. Valkunas, S. M. Bachilo, and G. R. Fleming, J. Phys. Chem. B 109, 15671 (2005).

[11] R.S. Lee, J. Gavillet, M. Lamy de la Chapelle, A. Loiseau, J.-L. Cochon, D. Pigache, J. Thibault, and F. Willaime, Phys. Rev. B 64, 121405(R) (2001)

[12] M. Rohlfing and S.G. Louie, Phys. Rev. B 62, 4927 (2000).

[13] W. Kohn and L.J. Sham, Phys. Rev. 140, A1133 (1965).

[14] M.S. Hybertsen and S.G. Louie, Phys. Rev. B 34, 5390 (1986).

[15] G. Strinati, Phys. Rev. B 295718 (1984).

[16] J. Ihm, A. Zunger, and M.L. Cohen, J. Phys. C 12, 4409 (1979).

[17] N. Troullier and J.L. Martins, Phys. Rev. 43, 1993 (1991).

[18] L. Kleinman and D.M. Bylander, Phys. Rev. Lett. 48, 1425 (1982).

[19] C.D. Spataru, S. Ismail-Beigi, L.X. Benedict and S.G. Louie, Appl. Phys. A 78, 1129 (2004).

[20] H. Ajiki and T. Ando, Physica B 201, 349 (1994).

[21] X. Blase, A. Rubio, S.G. Louie, M.L. Cohen, Phys. Rev. B 51, 6868 (1995).

[22] B. Arnaud, S. Lebegue, P. Rabiller, and M. Alouani, condmat/0503390.

[23] L. Wirtz, A. Marini, M. Gruning, and A. Rubio, submitted.

[24] G.Y. Guo and J.C. Lin, Phys. Rev. B 71, 165402 (2005).

[25] M.-F. Ng and R.Q. Zhang, Phys. Rev. B 69, 115417 (2004). 